\title{
Changes in the Influence of Atmospheric Humidity During Fatigue of an Aluminum Alloy
}

\author{
John A. Bennett
}

(December 12, 1963)

\begin{abstract}
The fatigue strength of 6061-T6 aluminum alloy specimens was found to be about 14 percent higher in a dry atmosphere than in a moist one. A series of experiments in which the humidity was changed after various numbers of cycles indicated that there was an initial perior during which the presence of water vapor had no effect on the total fatigue life. After this initial period, cracks developed and propagated much more rapidly in a moist atmosphere than in a dry one. The change in sensitivity to the environment is believed to result from rupture of the oxide film when the plastic strain becomes locally concentrated.
\end{abstract}

\section{Introduction}

In recent years there has been a revival of interest in the effects of environment on fatigue properties of metals. This interest has stemmed from several factors including (1) the need for accurate fatigue data for aircraft design purposes, so that the effect of mild corrosive agents such as water vapor can no longer be ignored; (2) the increased emphasis on mechanical tests in high vacuum resulting from the requirements of the space program; (3) the realization that the study of surface reactions can provide significant information regarding the mechanism of fatigue.

Results from a number of investigations have emphasized the importance of variations in humidity on the fatigue properties of aluminum alloys. Liu and Corten [1]* found that there was an inverse relation between the fatigue lives of aluminum alloy wires and the moisture content of the air during the tests. Although they made no effort to control the humidity, the variation in the laboratory over a period of months was sufficient to cause a difference of more than two to one in the mean fatigue life of specimens tested at a given stress amplitude. Coating their specimens with petroleum jelly reduced the variation, but did not result in as long a life as that of the specimens tested in dry air. Broom and Nicholson [2] used equipment in which a variety of atmospheres, including a high vacuum $\left(2 \times 10^{-6}\right.$ torr $)$, could be maintained around the specimen. They summarized the results of their tests on aluminum alloys by the conclusion that "the only constituent of normal air that significantly affects the fatigue life of age-hardened aluminum alloys is water vapour."

The results of the latter investigation provided some information on the relative influence of humidity on crack initiation and crack propagation, although there are certain inconsistencies. A few experiments in which the environment was changed

* Figures in brackets indicate the literature references at the end of this paper. during the fatigue test indicated that the total life was influenced only by the conditions during the latter part of the life, i.e., during crack propagation. However, the authors state that these results, taken in conjunction with the results of metallographic examination, "confirm that both crack initiation and growth are affected by the presence of water vapour."

The observation of gas evolution at the surface of specimens during fatigue testing appears to offer a new tool for studying the effects of environment, particularly on aluminum alloys. As reported by Holshouser and Bennett [3], this evolution can be seen readily, with specimens of the proper configuration, if a piece of transparent pressure-sensitive tape is applied to the surface of the test section. The gas, which is produced only during the latter part of the test, forms bubbles in the adhesive and lifts the tape from the surface. It was also observed that the tape had a marked effect on the fatigue life, presumably because it restricted the access of water vapor to the surface. In a few tests the specimens were stressed without tape for approximately half of their expected life, then the tape was applied; the total life in these tests was not significantly different from that obtained when the tape had been applied before stressing.

This result and the observations by Broome and Nicholson both suggested that there was a change in the nature of the reaction between the metal and its environment during the course of the fatigue process. In order to verify the occurrence of this change and to determine at what point it occurred, it appeared desirable to conduct a series of tests in which the environment was changed at different stages of the test. As the gas evolution phenomenon also indicated a change in the nature of surface reactions, there also appeared to be a need for experiments to determine if there was a relationship between the two changes. 


\section{Materials and Testing Technique}

The design of the test specimen was dictated to a large extent by the need for a configuration on which the evolution of gas could be observed. This requires that the fatigue cracks initiate in a surface to which pressure-sensitive tape can be firmly applied, and that the gas be unable to escape from under it. Many of the experiments reported in reference [3] were conducted with specimens of rectangular cross section which were stressed in torsion. These met the above requirement, but because of the extensive cracking that preceded fracture, it was difficult to define failure in a satisfactory manner. Also the probability of crack initiation is the same on two faces, one of which is difficult to inspect. For these reasons it was considered preferable to use specimens stressed in bending. The tape requirement can be met with a bend specimen if the cross section is thicker in the middle than it is at the edges so that the cracks do not start at the corners of the section. The design finally selected is shown in figure 1a.

Specimens were cut from a $120^{\circ}$ sector of tubing, and the reduced section was formed by a 2 -in. radius cut tangent to the inside surface of the tubing. They were stressed in cantilever bending, and it was found that all of the cracks started in the top surface if a mean tensile stress equal to $1 / 3$ of the stress amplitude was present in this surface. Tape could readily be applied to cover the test area, as it was singly curved with a radius of $3 / 4$ in.

The specimens were made from commercial 6061T6 aluminum alloy tubing. The selection of this alloy was based on the results of a previous investigation [4] in which the fatigue properties of 6061-T6 specimens were found to show less dispersion than either 2024-T4 or 7075-T6. The aging behavior of the material was determined with samples from each piece of tubing used for specimen blanks. The aging temperature used was $320{ }^{\circ} \mathrm{F} \quad\left(\begin{array}{ll}160 & { }^{\circ} \mathrm{C}\end{array}\right)$.
Maximum hardness was attained in about $30 \mathrm{hr}$ and no significant drop occurred in an additional $60 \mathrm{hr}$. Consequently, the specimen blanks were solution treated at $970{ }^{\circ} \mathrm{F}\left(520{ }^{\circ} \mathrm{C}\right)$ for $30 \mathrm{~min}$, quenched in water, and aged $48 \mathrm{hr}$ at $320^{\circ} \mathrm{F}\left(160{ }^{\circ} \mathrm{C}\right)$. This resulted in an average hardness of about 96 Rockwell F.

The specimens were machined and polished after heat treatment. Coarse circumferential polishing was carried out before the tubes were sectioned. After the specimens were machined they were polished longitudinally, the final operation being hand polishing with No. 600 paper. In order to have the abrasive conform to the curvature of the specimen adhesive-backed paper was mounted in a tube having an inside diameter of $1 \frac{5}{8}$ in. For the sake of uniformity, all specimens were given a brief final polish and inspection on the same day that they were to be tested. They were then measured as described below and finally they were washed in two successive baths of clean benzene. On removal from the second bath they were immediately inserted in the testing machine.

The minimum section of this specimen is a segment of a circle, so it was necessary to know the radius of curvature and thickness in order to calculate the minimum section modulus. Both of these dimensions were measured on each specimen, as it was found that the variations in radius were too large to be ignored.

The fatigue testing machine used for these experiments was of the constant load-amplitude type, the load being applied by an eccentric weight rotated $1800 \mathrm{rpm}$ by a synchronous motor. The alternating load was applied to the right end of the specimen as shown in figure $1 \mathrm{~b}$; any variation in the distance from the pivot pin to the minimum section of the specimen would, of course, cause a variation in the bending moment at that section. Consequently, in machining the specimens the holes at this end were used to position it for the transverse cut. In mounting the specimen in the machine, close-fitting screws

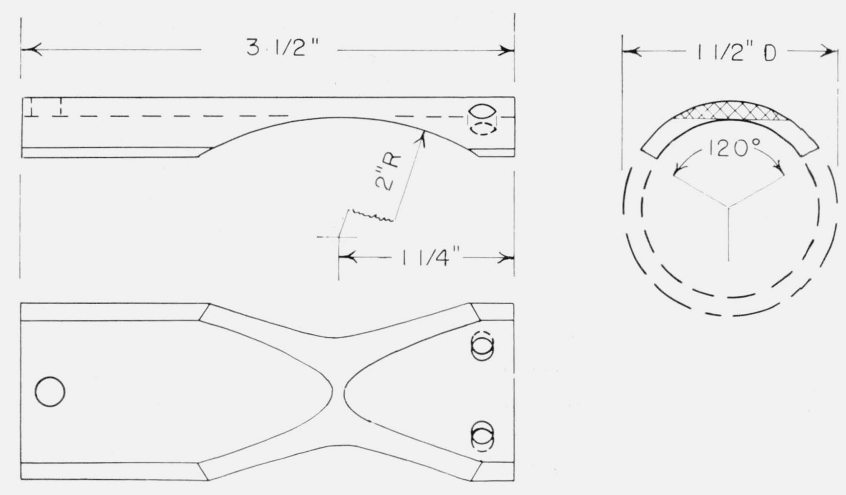

Figure 1a. Significant dimensions of the specimens used for reversed bending fatigue tests.

The wall thickness of the tubing was $1 / 8$ in. In the view at the right, the minimum section is cross-hatched. 


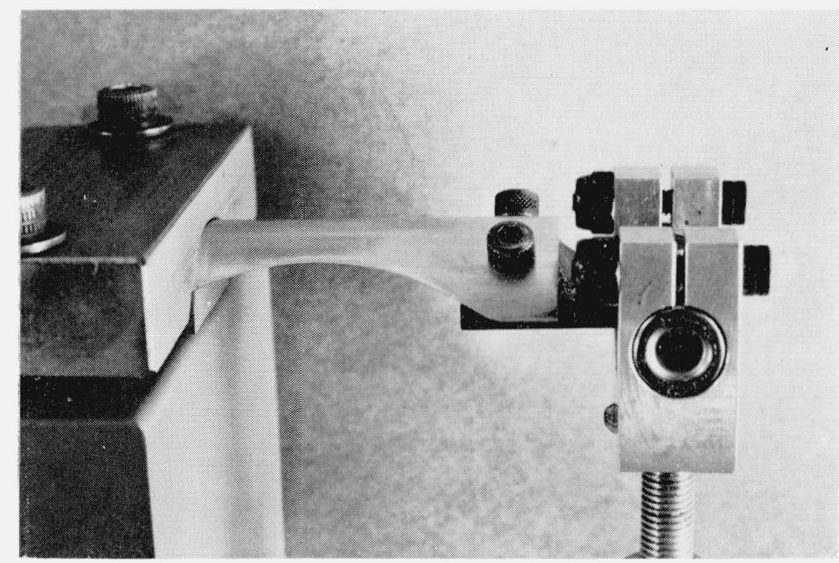

FIgURE 1b. Photograph showing a specimen mounted in the testing machine.

The alternating load is applied through the connecting rod and pivot assembly at the right. Full size.

were used in these holes, so that the moment arm was the same for all specimens. In all tests the mean load (directed so that the upper surface of the specimen was in tension) was one-third of the alternating load.

The entire testing machine was enclosed in a cabinet for humidity control. A motion picture camera was mounted above the specimen for taking time lapse exposures during tests of taped specimens, and a telescope passing through the top of the cabinet permitted viewing the test area at about $20 \times$ magnification. The humidity control was not automatic, so the actual values of relative humidity for the tests listed as "moist environment" varied from 85 to 95 percent while those for the "dry environment" ranged from less than 5 percent to 9 percent. These measurements were made with a commercial electrical hygrometer.

The experiments described in part 4 involved the application of transparent pressure-sensitive tape to the surface of the specimen. The tape used for these experiments was the ordinary cellophane-base type; one brand was used for all of the principal investigation, but supplementary tests indicated that a number of other brands, and even other types of tape, would make the gas evolution visible in much the same way.

A few preliminary tests were conducted with taped specimens in a low-humidity environment; no bubbling whatever was observed under these conditions. Consequently all of the "taped" tests described in part 4 were run in a moist atmosphere.

An adjustable contactor was used to stop the machine shortly before complete fracture of the specimen. Each of the contacts was a piece of platinum wire; one of these was mechanically and electrically connected to the connecting rod between the eccentric weight and the pivot pin. The other was on an insulated mount whose position relative to the moving contact could be adjusted by a screw from outside the cabinet. $\dot{A}$ dial indicator permitted reading the separation of the contacts to the nearest
0.0005 in. After a test was started the contactor was raised until it just touched the grounded wire at the bottom of the connecting rod stroke, as indicated by the action of an electronic relay. The contactor was then backed off the desired distance and the relay set to turn off the machine. It had been hoped that this device could be used to indicate the development of a small crack by setting the gap to a small value. However, the length of crack which resulted in cutoff was quite variable and consequently the technique was not useful in this investigation. The contactor did provide a reproducible method of stopping the test just short of fracture; it was set to a gap of 0.030 in., which resulted in a crack through about two-thirds of the section at cutoff. This preserved the original configuration of the specimen so it could be inspected to see if there were any surface features of significance. Also by completing the fracture manually after the specimen had been removed from the machine, the undamaged fracture surface was available for examination. This criterion of failure resulted in values of fatigue life only slightly smaller than would have been obtained at complete fracture.

It was found that considerable care was required in starting the machine to avoid excessive load. A variable autotransformer was used to increase the voltage on the motor very slowly until it pulled into synchronism. If this was not done the motor apparently overshot the synchronous speed, resulting in a few cycles of excessive deflection.

Although a total of three environments were used in this investigation (dry, moist, and taped), the environment was not changed more than once in any one test. Thus a total of nine types of test were conducted, as follows:

(1) Dry environment throughout

(2) Moist, then dry

(3) Dry, then moist

(4) Moist environment throughout

(5) Dry, then taped

(6) Moist, then taped

(7) Taped, then dry

(8) Taped, then moist

(9) Taped throughout.

The greatest emphasis was placed on tests which did not involve the use of tape (types one through four), and these will be discussed in the next section.

\subsection{Symbols}

$S_{A}$ - Stress amplitude.

$N_{1}$ - Number of cycles run in the first environment (in tests in which the environment was changed).

$N_{F}$ - Number of cycles to failure.

$N_{R}$-In the assumed relations such as those in figure 5 , the number of cycles to the break in the curve.

$N_{c}$-The estimated number of cycles to form a crack $0.1 \mathrm{~mm}$ long.

$N_{\mathrm{X}}$-For two-environment tests, the life to be expected on the basis of a linear interpolation.

$N_{p}-N_{F}$ minus $N_{c}$ 


\section{Results with Bare Specimens}

Conventional $S-N$ curves for specimens tested in both dry and moist environments are shown in figure 2. The points represent logarithmic means of the individual fatigue lives in all cases, the number of replicate tests being indicated on the graph. At 26.5 and $38 \mathrm{ksi}^{* *}$ the range of plus and minus one standard deviation is also shown. The average life of specimens tested in the dry environment was from two to three times that in high humidity. This difference corresponds to a difference in stress amplitude of approximately 14 percent throughout the range investigated. Although these environments were artificially produced, they were only slightly more divergent than conditions to be expected in a non-airconditioned laboratory during the course of a year. These results emphasize the possibility that humidity variations may have contributed to the scatter of results in many reported studies of fatigue.

Tests in which the environment was changed during the test were conducted at stress amplitudes of 26.5 and $38 \mathrm{ksi}$. The machine was stopped during the change, as this required as much as two hours in going from moist to dry atmospheres. The results of these tests are plotted in figures 3 and 4 . Graph "A" in each figure shows results for specimens that were run for $N_{1}$ cycles in dry atmosphere, then run to failure in a moist environment. The abscissas are the cycle ratios represented by $N_{1}$, that is the ratio of the number of cycles run in dry atmosphere to the mean life in that environment. The " $\mathrm{B}$ " graphs show similar results for specimens stressed first in a moist environment; the abscissas in these

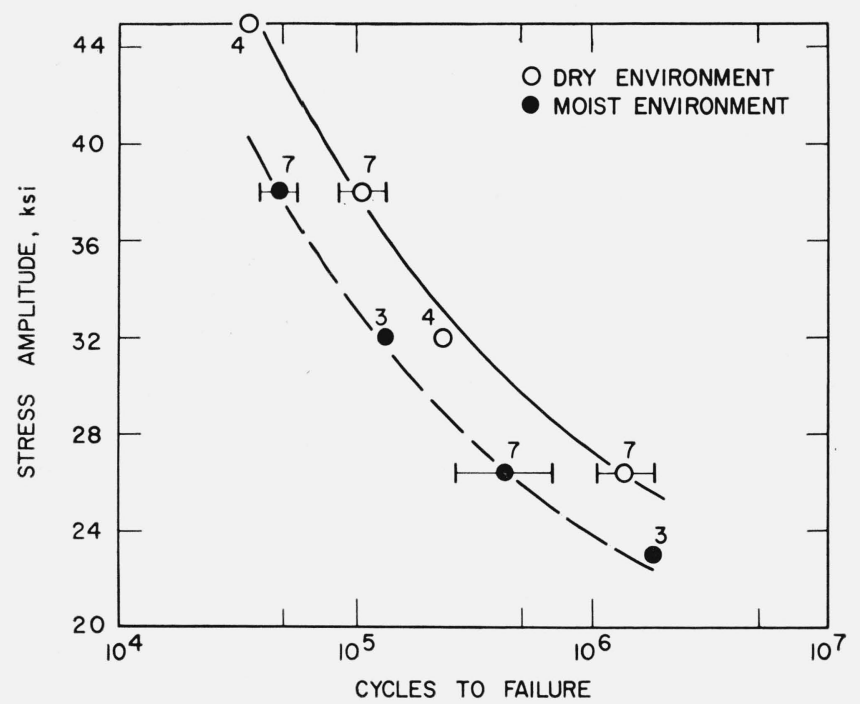

Figure 2. S-N curves for bare specimens in high- and lowhumidity environments.

In all tests the mean tensile stress on the test surface was $1 / 3$ of the stress amplitude. Numbers adjacent to points indicate the number of replicate tests; horizontal bars show the range of \pm one standard deviation.

** Thousands of pounds per square inch.
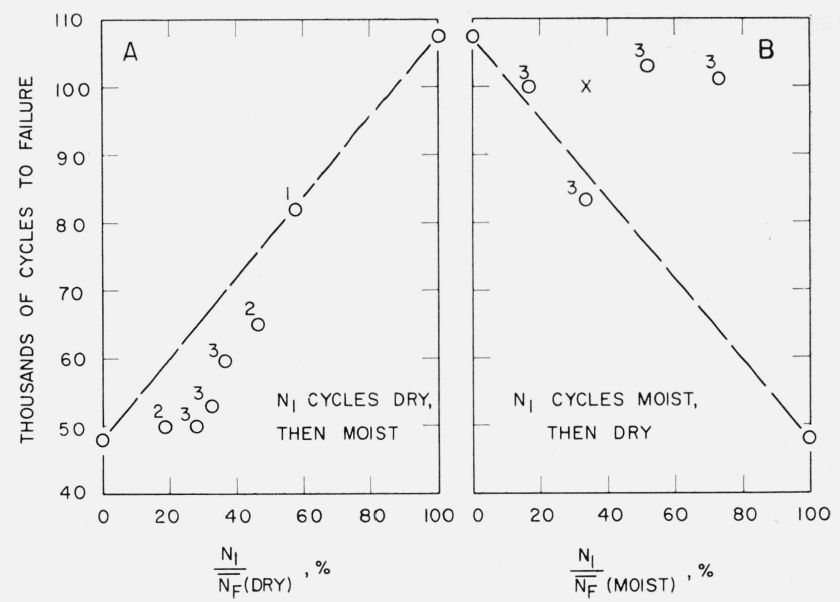

FiguRE 3. Results of two-environment tests at $\mathrm{S}_{A}=26.5 \mathrm{ksi}$.
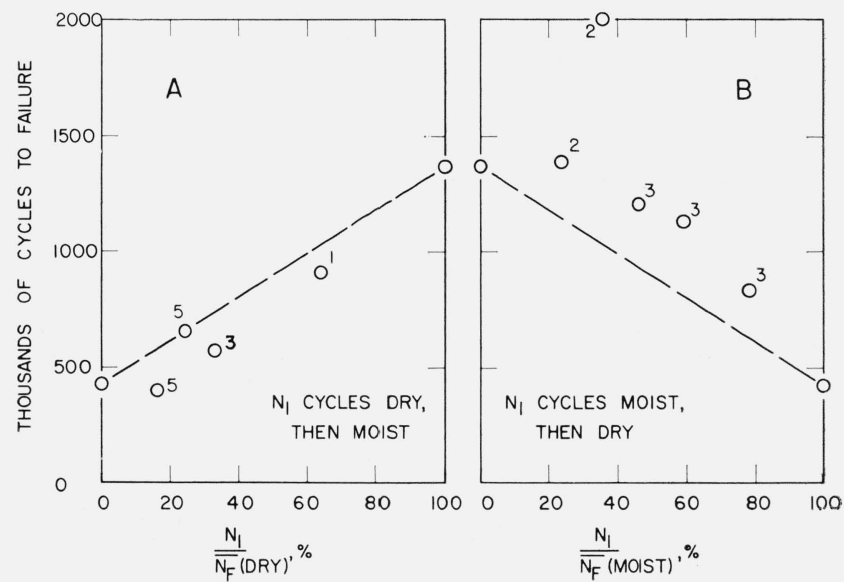

FIgURE 4. Results of two-environment tests at $\mathrm{S}_{\boldsymbol{A}}=38 \mathrm{ksi}$.

graphs represent cycle ratios based on the mean life in a high-humidity atmosphere. The end points for the two graphs in each figure are the same, as they are the number of cycles to failure for specimens tested in a single environment.

As in all fatigue testing, the dispersion of the results is troublesome in attempting to determine the true nature of the behavior indicated by the data. In most cases the logarithmic mean values shown in figures 3 and 4 are thought to give reasonably good indications of the true central value; however, the point at 33 percent in graph "B" of figure 4 is probably unduly influenced by one very low result. The mean value of the other two results in this group is shown by the cross, and this appears much more consistent with the rest of the data.

It is apparent from figures 3 and 4 that the fatigue life is not a linear function of the cycle ratio in the first environment; with the exception of the point discussed above, all of the points in the "A" graphs are below the straight line connecting the mean 
single-environment values, and all of the points in the "B" graphs are above this line. The divergence is particularly large in tests run first in the moist environment; at a stress amplitude of $38 \mathrm{ksi}$ specimens stressed for more than 70 percent of their expected life in this environment had a mean life only a few percent less than that of specimens tested throughout in a dry atmosphere. Likewise at 26.5 ksi the initial stressing in moist environment seemed to have little effect on the total life; actually the specimen that ran the longest of all those tested at this stress amplitude was one that had been stressed in moist atmosphere for 30 percent of its expected life.

Even though the dispersion of the data was not thought to be excessive, it was large enough to make it difficult to fit trend lines directly. Instead it was considered more informative to make the simplest possible assumptions regarding the behavior of the specimens, then determine if the observed data are consistent with this hypothetical behavior. As the data are clearly not consistent with the linear relation in figures 3 and 4 , it might be assumed that the relation is actually two straight lines, the first one horizontal. This would mean that the total life is not influenced by the environment during the early part of the test. Such hypothetical relationships for the tests at $38 \mathrm{ksi}$ are shown in figure 5. If the humidity has no effect during the early part of the test, then the break in the curves must come at the same number of cycles for both environments. This number, indicated by $N_{R}$ on figure 5 , was determined by trying different values until the best fit for both sets of data was found.

The data were compared with the hypothetical relations by plotting the deviations from the linear dependence of $N_{F}$ on $N_{1}$. The life to be expected

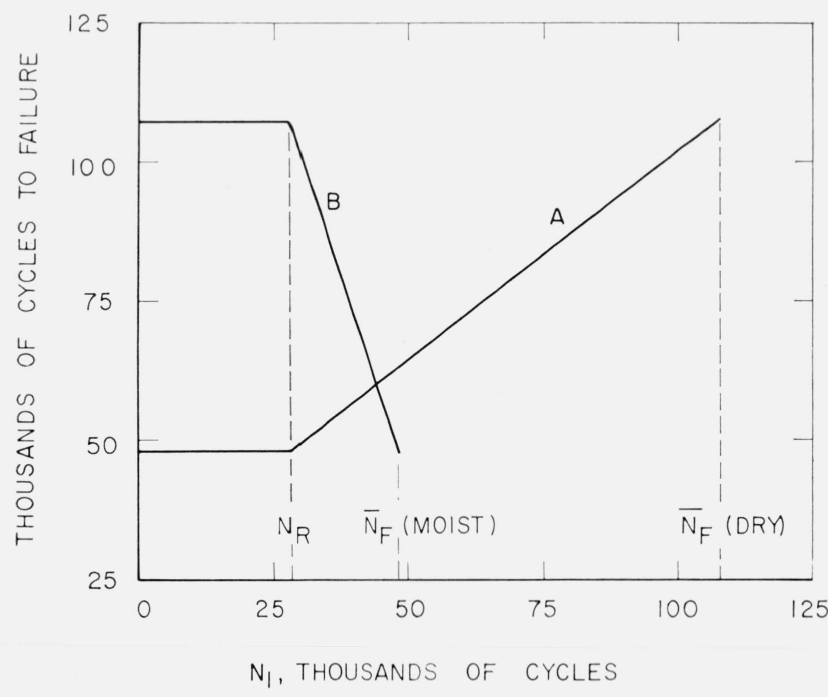

Figure 5. Assumed relationship between $\mathrm{N}_{1}$ and $\mathrm{N}_{F}$, for twoenvironmental tests at $\mathrm{S}_{A}=38 \mathrm{ksi}$.

Curve A, dry environment first; curve B, moist environment first.

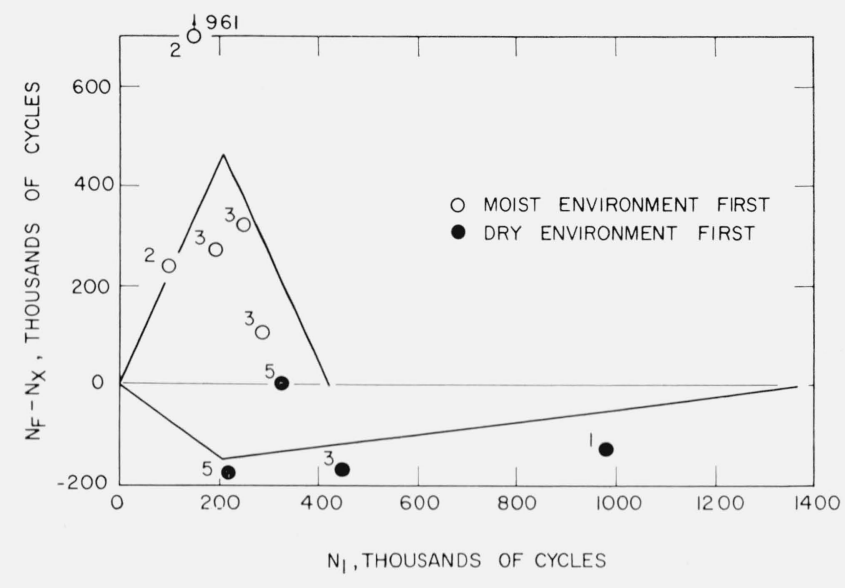

Figure 6. Difference between observed fatigue life and that expected on the basis of a linear dependence on $\mathrm{N}_{1}$, tests at $\mathrm{S}_{A}=26.5 \mathrm{ksi}$.

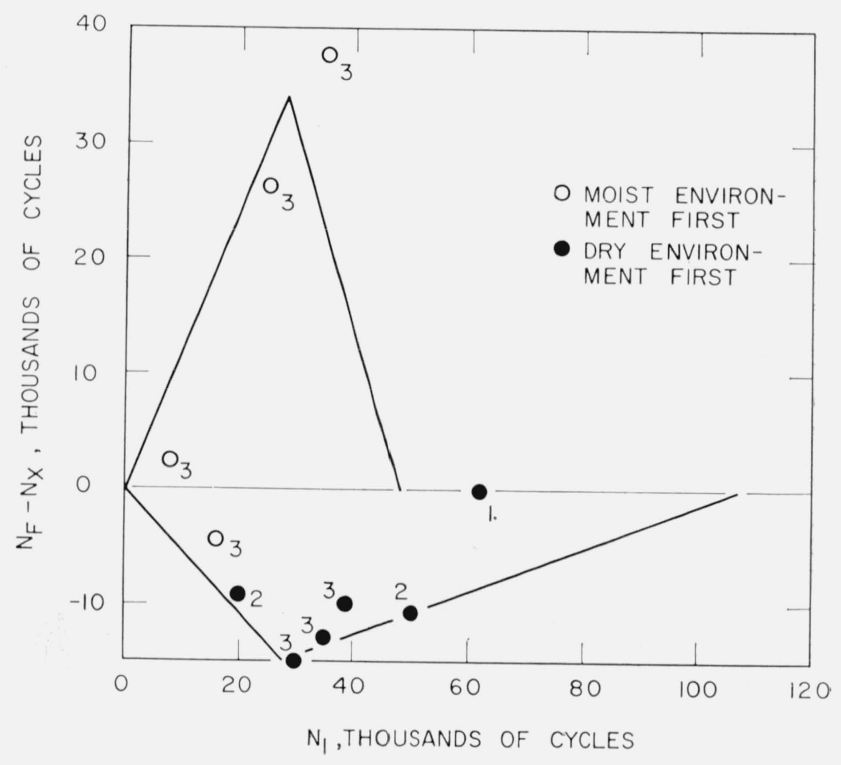

Figure 7. Data similar to figure 6 , for tests at $\mathrm{S}_{A}=38 \mathrm{ksi}$.

on the basis of the straight lines in figures 3 and 4 is designated $N_{x}$; figures 6 and 7 show the differences between the observed mean $N_{F}$ and $N_{x}$ for the same values of $N_{1}$. The solid lines on these figures represent the values to be expected on the basis of the assumptions of figure 5, i.e., that the total life is influenced by the environment only after $N_{R}$. Although many of the points are far from these lines, the deviations are both positive and negative, and the sums of the deviations in each figure are much less than the deviations from a linear relation. Thus the assumptions shown in figure 5 appear to be consistent with the results; in view of the dispersion of the data it is probably not worthwhile to see if a more complicated hypothesis would provide a better fit. Results of the tests with bare specimens are summarized in table 1. 
TABLE 1. Results of tests with bare specimens

\begin{tabular}{|c|c|c|c|}
\hline Stress amplitude & $N_{R}$ & Atmosphere & $N_{F}$ \\
\hline $\begin{array}{l}26.5 \mathrm{ksi} \\
38 \ldots\end{array}$ & $\begin{array}{l}210 \times 10^{3} \\
28\end{array}$ & $\begin{array}{l}\text { Dry } \\
\text { Moist } \\
\text { Dry } \\
\text { Moist }\end{array}$ & $\begin{array}{l}1,369 \times 10^{3} \\
423 \\
107.4 \\
48.1\end{array}$ \\
\hline
\end{tabular}

\section{Results with Taped Specimens}

Figure 8 shows enlargements of a few of the timelapse exposures taken of a taped specimen. Prior to the application of the tape this specimen had been subjected to $900 \times 10^{3}$ cycles at a stress amplitude of $26.5 \mathrm{ksi}$ in a dry environment, but the development of bubbles under the tape is typical of all the specimens photographed. No change is observed during the early part of the test, but once the first bubble appears the gas continues to be evolved until the end of the test. In the test of figure 8 the first bubble appeared at $1217 \times 10^{3}$ cycles and failure occurred at $1455 \times 10^{3}$ cycles.

In the torsion experiments reported in reference [3] it was found that gas evolution started before definite fatigue cracks could be detected either by surface examination or by metallographic examination of the cross section of the specimen. In the current experiments, however, when tests of taped specimens were stopped as soon as gas evolution was observed, it was always possible to see small cracks under the bubbles. If the tests were monitored carefully, the cracks were only a few tenths of a

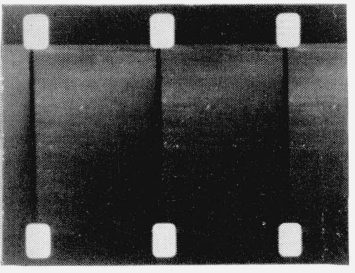

a

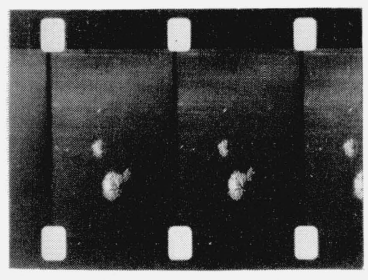

C

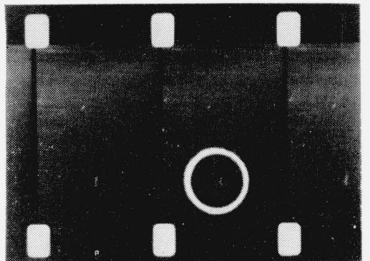

b

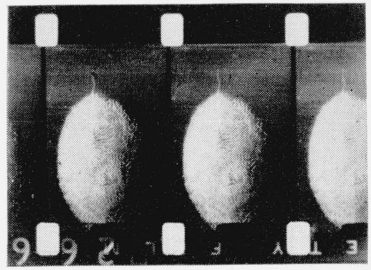

d
FIGURE 8. Time lapse exposures taken during test of a taped specimen at $\mathrm{S}_{A}=26.5 \mathrm{ksi}$.

These pictures show the surface (magnification $1.5 \times$ ) at the following numbers of cycles:

$$
\begin{array}{ll}
\text { (a) }-1200 \times 10^{3} & \text { (b) }-1280 \times 10^{3} \\
\text { (c) }-1350 & \text { (d) }-1455
\end{array}
$$

$N_{c}$ was determined to be $1217 \times 10^{3}$ cycles, $N_{F}$ was $1455 \times 10^{3}$.

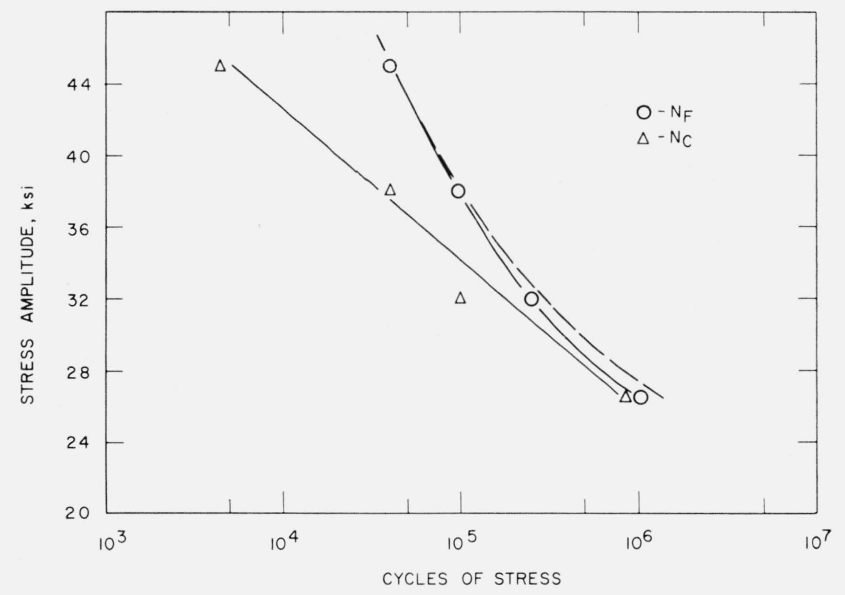

FIgURE 9. S-N curves for specimens tested with tape on the surface in moist atmosphere.

The dashed line is the curve from figure 2 for bare specimens in low humidity.

millimeter in length and could be observed only on a metallographic microscope. The first appearance of a bubble on the motion picture film provided a convenient and sensitive means of separating the fatigue life into two stages. It was estimated that the crack length at this point in a test was about $0.1 \mathrm{~mm}$. Consequently, in tests of taped specimens, $N_{c}$ was taken as the number of cycles at which gas evolution was first observed on the films. In order to obtain values of $N_{c}$ for the other two environmental conditions (bare specimens), a series of two-environment tests was conducted in which tape was applied or removed.

Figure 9 shows the $S-N$ curves for specimens covered with pressure-sensitive tape throughout the test. The open circles represent the logarithmic mean life to failure from tests of three or four specimens at each stress level. The triangular points are median values of $N_{c}$, as defined above. The dashed line is taken from figure 2 and represents the data for bare specimens tested in a dry environment. The results with taped specimens differed significantly from this curve only at the low stress end.

Four types of test were conducted in order to estimate the values of $N_{c}$ for bare specimens; these are listed as types 5 through 8 in part 2 above. In types 5 and 6 , the bare specimens were stressed for $N_{1}$ cycles, then were taped for the remainder of the test. If $N_{1}$ was more than $N_{c}$, gas evolution was observed immediately on restarting the testing machine. If $N_{1}$ was less than $N_{c}$, the time lapse pictures could be used to determine the actual value of $N_{c}$ for the specimen. A sequential procedure was used for these tests in an effort to assign values of $N_{1}$ close to $N_{c}$.

The tests of types 7 and 8 (initially taped) were monitored as well as being photographed, and the test was stopped as soon as definite bubbles were seen. The specimen was removed from the machine for cleaning, then replaced and run to failure in either a dry or moist atmosphere. 
The purpose of all types of tests involving the use of tape was to provide estimates of $N_{c}$ for bare specimens to be compared with the values of $N_{R}$. The tests with initially bare specimens bracketed $N_{c}$, but because of the dispersion of the value from specimen to specimen this was a very inefficient type of determination. The results of tests in which the specimens were initially taped gave values of $N_{F}-N_{c}$ (designated $N_{p}$ ) for bare specimens. The difference between these values and the appropriate mean life $\left(\bar{N}_{F}\right)$ listed in table 1 gave the second estimate of $N_{c}$ for each condition. Both of these determinations involve some approximations that cannot be rigorously justified, so the values given in table 2 must be considered as only semi-quantitative.

TABLE 2. Results from two-environment tests using tape

\begin{tabular}{|c|c|c|c|c|}
\hline Stress amplitude & Atmosphere & $N_{c}^{(1)}$ & $N_{p}$ & $N_{c}^{(2)}$ \\
\hline $\begin{array}{l}26.5 \mathrm{ksi} \\
26.5\end{array}$ & $\begin{array}{l}\text { Dry } \\
\text { Moist }\end{array}$ & $\frac{1,100 \times 10^{3}}{200}$ & $\begin{array}{l}310 \times 10^{3} \\
120\end{array}$ & $\begin{array}{l}1,060 \times 10^{3} \\
300\end{array}$ \\
\hline $\begin{array}{l}38 \\
38 \\
38\end{array}$ & $\begin{array}{l}\text { Dry } \\
\text { Moist_- }\end{array}$ & $\begin{array}{l}37 \\
20\end{array}$ & $\begin{array}{l}85 \\
25\end{array}$ & $\begin{array}{l}22 \\
23\end{array}$ \\
\hline
\end{tabular}

(1) From tests with initially bare specimens.

(2) $N_{c}=\bar{N}_{P}-N_{p}$.

In attempting to arrive at a "best value" of $N_{c}$ from the above data, it is not suitable to average the values in columns 3 and 5 of table 2. For example, the number 22 in column 5 is the difference of two much larger numbers, so that its uncertainty is greater than that of the other result for the same conditions. Consideration of such factors leads to the estimates listed in table 3, of the most probable values of $N_{c}$. Data from table 1 and from figure 9 are included for comparison.

TABLE 3. Summary of test results

\begin{tabular}{|c|c|c|c|c|}
\hline Stress amplitude & $N_{R}$ & Atmosphere & $N_{0}$ & $N_{F}$ \\
\hline $26.5 \mathrm{ksi}$ & $210 \times 10^{3}$ & $\begin{array}{l}\text { Dry } \\
\text { Moist } \\
\text { Taped.. }\end{array}$ & $\begin{array}{l}1,100 \times 10^{3} \\
250 \\
850\end{array}$ & $\begin{array}{l}1,369 \times 10^{3} \\
423 \\
1,033\end{array}$ \\
\hline 38 & 28 & $\begin{array}{l}\text { Dry } \\
\text { Moist-. } \\
\text { Taped }\end{array}$ & $\begin{array}{l}35 \\
22 \\
40\end{array}$ & $\begin{array}{r}107.4 \\
48.1 \\
97.3\end{array}$ \\
\hline
\end{tabular}

Although $N_{R}$ and $N_{c}$ are given to two significant figures, it must be remembered that they are based on data that show a large dispersion. It is difficult to conceive of a mechanism that would result in a value of $N_{R}$ larger than $N_{c}$, as shown for one condition, and this is probably not actually the case. On the other hand it is believed that the magnitudes are approximately correct and that, for example, the difference between $N_{R}$ and $N_{c}$ in the first line of the table is certainly significant. Thus the data indicate that $N_{R}$ represents a discontinuity in the fatigue process which is distinct from the discontinuity represented by the development of the first crack.

These results emphasize the magnitude of the influence that water vapor has on the fatigue behavior of this alloy, since the entire effect takes place between $N_{R}$ and $N_{F}$. At 26.5 ksi this portion of the life is five times as long in a dry atmosphere as it is in a moist one, and the ratio is four to one at $S_{A}=38 \mathrm{ksi}$. Another interesting point brought out by these results is that at a stress amplitude of $38 \mathrm{ksi}$, the life in low-humidity air of a specimen containing a visible fatigue crack is greater than the total life of a specimen in a moist environment.

\section{Discussion}

The evolution of gas makes it clear that a chemical reaction is taking place during crack propagation in this aluminum alloy. It was deduced in reference [3] that the gas is hydrogen resulting from a reaction between water and the fresh metal surface exposed during severe local deformation or crack propagation. This conclusion is substantiated by the observation that no gas evolution is observed when the test is conducted in a low-humidity atmosphere.

Other observations made during this investigation provide some further clarification of the conditions under which the gas evolution can be observed by the development of bubbles under transparent pressure-sensitive tape. As mentioned earlier, the bubbles on these bend specimens were always associated with visible cracks whereas in the earlier work on torsion specimens of 5052-H34 alloy gas evolution was observed before cracks could be detected. This suggests that the development of bubbles depends on the rate of gas production; if this rate is low the hydrogen can diffuse out through the tape before the pressure builds up enough to form a bubble. It would be expected that the rate would be affected by the area of fresh metal being exposed and by the supply of moisture available at the metal surface. Apparently the oxide film was ruptured over a greater area before crack formation in the torsion tests of the cold-worked alloy than in the bend tests of the precipitation-hardened material. Likewise, when the supply of water is reduced by running the test in low humidity, the rate of reaction may not be sufficient to result in bubble formation. Thus the point at which the first bubble is observed probably does not represent a discontinuity in the fatigue process but must be interpreted as the point at which the rate of gas evolution reaches a critical value.

On the other hand, the results from two-environment tests of bare specimens indicate that there is a period during the early part of the fatigue process when the humidity of the environment has no effect. As there is a marked effect after this initial period, there appears to be a definite point where the nature of the surface is changed so that a reaction with the environment can take place. The data of table 3 indicate that this point is significantly earlier in the fatigue life than the start of a detectable crack, at least for specimens run in a dry environment. At both stress levels the values of $N_{R}$ and $N_{\mathrm{c}}$ for the moist environment are nearly equal, while for the dry tests there is a large difference in the two values at $26.5 \mathrm{ksi}$, and the difference at $38 \mathrm{ksi}$ is probably 
significant. These results suggest that the greatest effect of the reaction with water may occur between $N_{R}$ and $N_{\mathrm{c}}$; in moist environment a crack forms very soon after $N_{R}$ whereas this process takes much longer under low-humidity conditions.

The data of figure 9 show that the tape limits the access of water to the surface of the metal to such an extent that the fatigue behavior is nearly the same as that of bare specimens in a dry environment. The evolution of gas under the tape shows that some moisture is reaching the surface, so the results of figure 9 indicate that the damaging effect of moisture depends on a fairly high rate of reaction. This is also shown by the fact that the $S-N$ curve for taped specimens coincides with that for dry specimens at the high-stress end, but is significantly lower at the lower stress amplitudes where more time is available for water to diffuse through the tape.

The results of this investigation appear to be consistent with the view that the humidity starts to affect the fatigue behavior when the deformation becomes localized, thus breaking the oxide film. During the early part of the fatigue life the deformation is apparently sufficiently uniform so that the film remains intact and no reaction with the environment occurs. Once the film is ruptured, cracks develop rapidly if moisture is available, much more slowly if the supply is limited. The reason for this effect is not clear, but it apparently involves the oxidation of the aluminum with the liberation of hydrogen. One possibility that has been suggested
[5] is that if the fresh surface formed during slip in one direction is oxidized, reverse slip cannot occur on the same plane, so the damage from repeated slip is cumulative. If the freshly formed surface is not oxidized, the slip can be reversed on the same plane, thus canceling much of the effect of the forward slip.

\section{Miscellaneous Observations}

A number of observations that were not related to the primary purpose of the investigation are considered to be of some general interest.

\subsection{Amount of Shear Plane Cracking}

The fracture surfaces of all specimens were examined after testing. As expected, the initial plane of the cracks appeared to make an angle of approximately $45^{\circ}$ with the stress direction, but at a later stage the fracture surface was generally perpendicular to this direction. All of the fractures were irregular, but there seemed to be a definite influence of stress amplitude and environment on the amount of shear plane cracking. As shown in figure 10, the specimens tested at high stress amplitudes and in dry atmospheres were apt to have a large area of shear plane fracture, while in those tested at low stress or high humidity the shear areas were found only close to the origin. (The section shown in figure $10 \mathrm{~b}$ did not pass close enough to the origin to intersect any such areas.) These

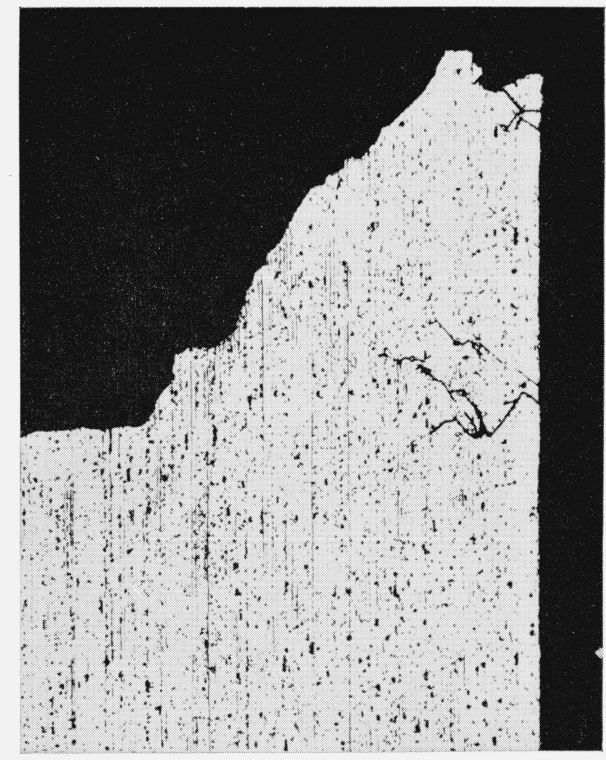

a

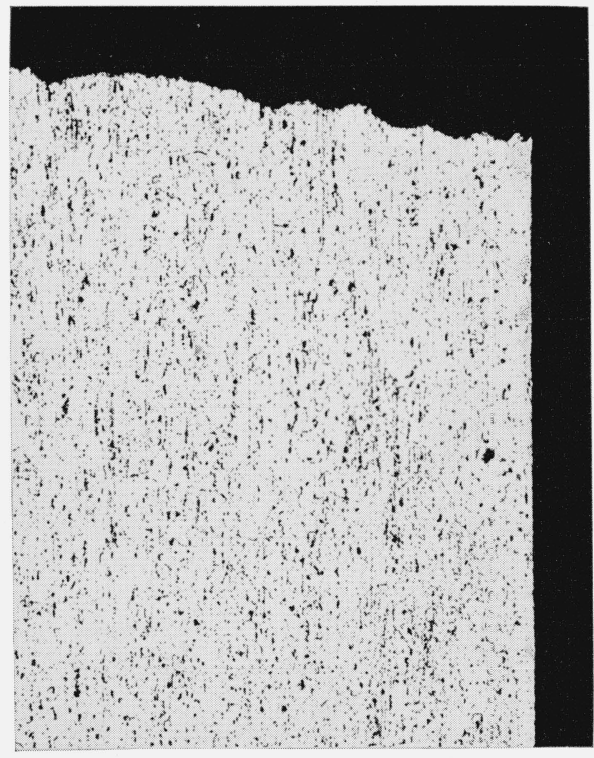

b

FiguRE 10. Cross sections of fractured specimens intersecting the fracture (top) and the upper surface (right). $\times 50$.

a. $S_{A}=45 \mathrm{ksi}$, tape on surface throughout test.

b. $S_{A}=22 \mathrm{ksi}$, moist environment. 
results are contrary to those reported by Forsyth, Stubbington, and Clark [6]; undoubtedly there are other factors, such as mean load, that have an influence.

\subsection{Color on Fracture Surface}

With many of the specimens, it was observed that certain areas of the fracture surface produce bright colors when obliquely illuminated with a concentrated light source. By mounting the fracture surfaces in the fractographic specimen mount described in reference [7], the colored areas could be observed at 50 to $150 \times$ on a metallograph. The colors appeared brightest when the specimen was illuminated directly (rather than through the vertical illuminator) with an angle of about $45^{\circ}$ between the incident light and the axis of the microscope. The individual areas showing color were not more than a few tenths of a millimeter in extent, but in some parts of the fracture a considerable proportion of the total area was made up of these colored spots. In all cases the greatest amount of color was observed in the portion of the fracture where the crack had propagated rapidly. No color was observed on the static portion of the fracture nor on specimens that had been broken in low humidity at relatively low stress levels (conditions resulting in slow crack propagation).

The colors appeared to be spectrum colors rather than subtractive colors such as those observed due to interference films. Changing the angle between the surface and the direction of viewing resulted in a change in the observed color. When the colorproducing areas were examined at higher magnification with vertical illumination, they were always found to contain well-defined striations on the sur- face. This suggested that the observed colors may be due to diffraction from these striated areas.

Replicas were made of a fracture surface that showed colored areas, and the electron micrographs from these replicas (fig. 11) made it evident that the striations are sufficiently well defined to act as a diffraction grating. The striation spacings measured on several micrographs ranged from 0.1 to $1.3 \mu$. As spacings in this range are consistent with low-order diffraction of visible light, this is probably the correct explanation for the observed color.

There is considerable current interest in the topography of fatigue fracture surfaces, as evidenced, for example, by the excellent investigations of Forsyth and others at the Royal Aircraft Establishment [8]. It appears that the diffraction grating effect might provide a method of measuring striation spacings that could be used for areas that are not accessible for direct observation at high magnifications.

\subsection{Usefulness of the Gas Evolution Phenomenon in Fatigue Investigations}

The time lapse motion pictures of taped specimens that were taken during this investigation have suggested a number of ways in which the phenomenon of gas evolution might be of use in fatigue studies. The bubble formation under the tape provides an extremely convenient and sensitive crack detection device. As such it has the great advantage that it is easily photographed; this permits both accurate location of the crack and accurate determination of the number of cycles at which the first bubble formed. It appears that these capabilities should be of value, for example, in statistical studies where it is desirable to know the actual stress at the point
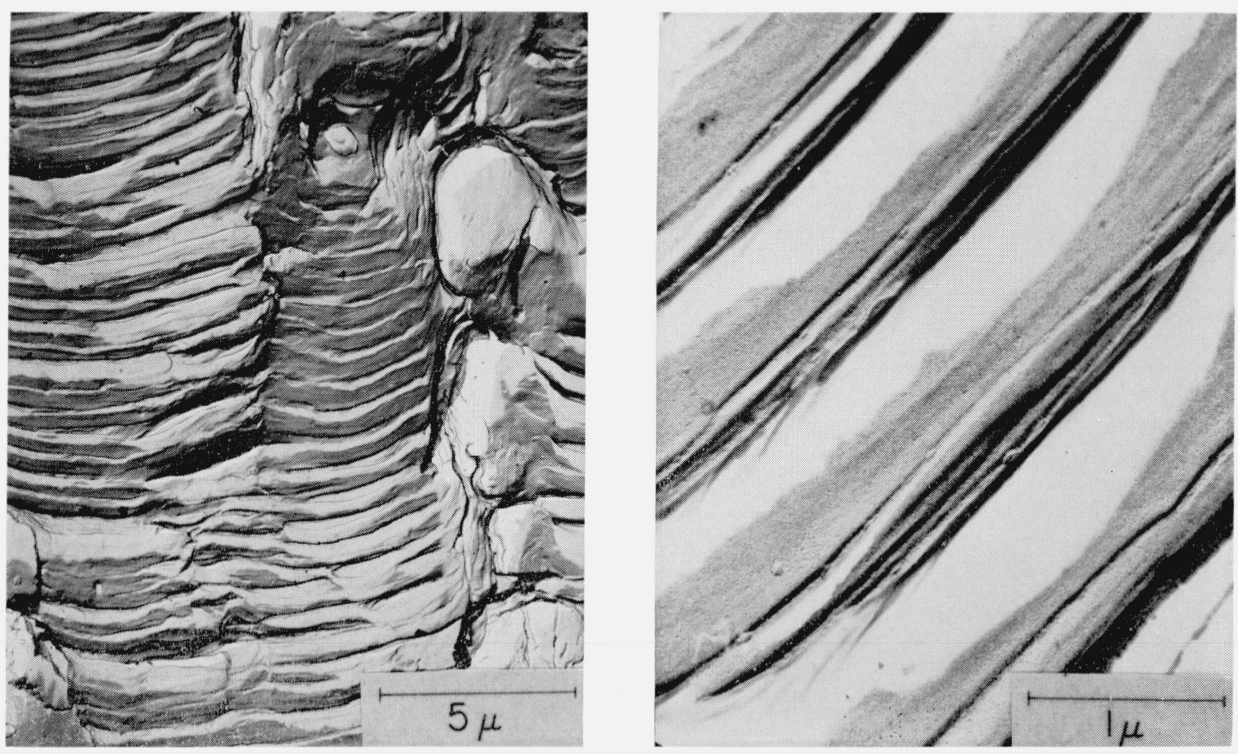

FIgURE 11. Typical electron micrographs of a fractured surface on which colored areas were observed. 
of crack initiation, or in the many cases when it is helpful to separate the fatigue life into periods of crack initiation and crack propagation.

\section{Conclusions}

Results of reversed bending fatigue tests on specimens of 6061-T6 aluminum alloy appear to justify the following conclusions:

1. The fatigue life in an environment of about 5 percent relative humidity was two to three times as long as that in high humidity. This difference corresponds to a difference in stress amplitude of about 14 percent throughout the range investigated.

2 . The effect of humidity on the number of cycles to failure was much more pronounced during the latter part of the test than during the initial portion.

3 . The data are consistent with the view that there was an initial period during which the humidity had no effect on the fatigue behavior of this alloy.

4. The portion of the test during which the humidity had no effect was significantly shorter than the number of cycles required to form a detectable crack in a low humidity environment. In a moist atmosphere cracks apparently developed very soon after this initial period.

5. At a stress amplitude of $38 \mathrm{ksi}$, the mean life of specimens containing a small fatigue crack, tested in a dry environment, was greater than the total life of specimens tested in a moist atmosphere.

6. Gas evolution under pressure-sensitive tape was observed only after visible fatigue cracks were present.

7. The development of bubbles under transparent tape provided a convenient and sensitive method of detecting small cracks.

8. No gas evolution was observed when the test was conducted in a low-humidity environment.

9. The extent of shear plane cracking increased with increasing stress amplitude and with decreasing humidity.

10. The results seem to be consistent with the view that during the early part of the fatigue process the deformation is fairly uniform, so that the local strain is nowhere sufficient to break the oxide film. As stressing proceeds, the deformation becomes localized, breaking the film so that the fresh metal surface is exposed to the environment.

11. The striations on some of the fracture surfaces were sufficiently pronounced to produce bright colors by diffraction.

There does not appear to be any reason to believe that the results of this investigation are unique to the alloy investigated; it is probable that they are typical of age-hardened aluminum alloys in general.

\section{References}

[1] H. W. Liu and H. T. Corten, Fatigue damage under varying stress amplitudes, NASA TN D-647, Nov. 1960.

[2] T. Broom and A. Nicholson, Atmospheric corrosionfatigue of age-hardened aluminum alloys, J. Inst. Metals 89, 183, 1961.

[3] W. L. Holshouser and J. A. Bennett, Gas evolution from metal surfaces during fatigue stressing, Proc. ASTM 62, 683, 1962.

[4] J. A. Bennett and J. G. Weinberg, Fatigue notch sensitivity of some aluminum alloys, J. Res. NBS 52, 235, $1954, \mathrm{RP} 2495$.

[5] N. J. Wadsworth, Effect of environment on metal fatigue, Internal Stresses and Fatigue in Metals, p. 382, Elsevier Publ. Co., 1959.

[6] P. J. E. Forsyth, C. A. Stubbington, and D. Clark, Cleavage facets observed on fatigue-fracture surfaces in an aluminum alloy, J. Inst. Metals 90, 238, Feb. 1962.

[7] D. B. Ballard and J. A. Bennett, A magnetic specimen mount for fractography, Metal Progr. 74, 114, Nov. 1954.

[8] P. J. E. Forsyth and C. A. Stubbington, Some observations on the initiation and growth of fatigue cracks in an Aluminum-7.5 zinc-2.5 magnesium alloy, Royal Aircraft Establishment Tech. Note No. Met-Phys 344, July 1962 .

(Paper 68C2-155) 